\title{
The effect of Aurora kinases on cell proliferation, cell cycle regulation and metastasis in renal cell carcinoma
}

\author{
YAN LI ${ }^{1}$, WANQI ZHOU ${ }^{1}$, LANGLI WEI ${ }^{1}$, JING JIN $^{1}$, KE TANG $^{1}$, \\ CHAO LI ${ }^{1}$, BIN TEAN TEH ${ }^{2}$ and XIAOGUANG $\mathrm{CHEN}^{1}$ \\ ${ }^{1}$ State Key Laboratory of Bioactive Substances and Functions of Natural Medicines, Department of Pharmacology, \\ Institute of Materia Medica, Chinese Academy of Medical Sciences and Peking Union Medical College, \\ Beijing 100050, P.R. China; ${ }^{2}$ NCCS-VARI Translational Research Laboratory, \\ National Cancer Center, Singapore 169610, Republic of Singapore
}

Received June 6, 2012; Accepted August 2, 2012

DOI: 10.3892/ijo.2012.1633

\begin{abstract}
Aurora kinases have been shown to be involved in the regulation of the cell cycle and are related to tumor progression. This suggests the possibility that they can serve as new anticancer targets for tumor treatment. However, the important roles that Aurora kinases and their signaling pathway play in renal cell carcinoma (RCC) are not fully understood and addressed to date. In this study, we aimed to address these questions. We observed that downregulation of Aurora kinases induced by AurA miRNA, AurB miRNA or VX680 could inhibit proliferation and metastasis, induce $\mathrm{G} 2 / \mathrm{M}$ phase arrest in clear cell renal cell carcinoma cells and exert antitumor activity in an SN12C xenograft model. We also show that either silencing of Aurora kinases or treating the cells with VX680 could downregulate the expression of $\mathrm{cdc} 25 \mathrm{c}$ and cyclin $\mathrm{B} / \mathrm{cdc} 2$, upregulate the expression of p-cdc2 (Tyr15) via blocking the activity of ERK. All these changes may contribute to inhibition of proliferation, metastasis and G2/M arrest in ccRCC. In summary, we proved that both Aurora kinases A and B are key elements of tumor growth regulation, and inhibition of Aurora kinases may contribute to blocking ccRCC progression. We conclude that Aurora kinases could be potential therapeutic targets in the management of renal cell carcinoma.
\end{abstract}

Correspondence to: Professor Xiaoguang Chen, State Key Laboratory of Bioactive Substances and Functions of Natural Medicines, Department of Pharmacology, Institute of Materia Medica, Chinese Academy of Medical Sciences and Peking Union Medical College, 1 Xian Nong Tan Street, Beijing, 100050, P.R. China

E-mail: chxg@imm.ac.cn

Dr Bin Tean Teh, NCCS-VARI Translational Research Laboratory, National Cancer Center, 11 Hospital Drive, Singapore 169610, Republic of Singapore

E-mail: teh.bin.tean@singhealth.com.sg

Key words: Aurora kinases, clear-cell renal cell carcinoma, proliferation, metastasis, G2/M arrest, p-ERK

\section{Introduction}

Renal cell carcinoma (RCC) is the most common type of kidney cancer in adults and its incidence has increased consistently for the past 20 years (1). The main curative treatment for RCC is surgery, but the tumor often has recurrence or the patients have metastatic disease after surgery (2). Therefore, the identification of effective and specific novel targets for early diagnosis and treatment are necessary. Insights into the biology of clear-cell renal cell carcinoma (ccRCC) have identified multiple pathways associated with the pathogenesis and progression of this cancer, leading to the development of a number of agents targeting these signaling pathways, which include the tyrosine kinase inhibitors sorafenib, sunitinib and pazopanib, the monoclonal antibody bevacizumab, and the mTOR inhibitors temsirolimus and everolimus (3).

Aurora kinases are a family of conserved mitotic regulators consisting of Aurora kinase A, B and C in humans (4). The activities of Aurora kinases depends on autophosphorylation of Thr288 in the activation loop of Aurora A and phosphorylation Thr232 in Aurora B. Aurora kinase A and B have been well characterized to be important players in mitosis (5). They are overexpressed in tumors such as prostate cancer, esophageal squamous cell carcinoma, and head and neck squamous cell carcinoma, and are shown to be positively correlated with chromosomal instability and clinical aggressiveness in the malignancies (6). Along with cyclins and cyclin-dependent kinases, Aurora kinases have been reported to link to G2/M transition of cell cycle (7). Moreover, several reports have demonstrated that Aurora kinases interact with many important cellular proteins related to cell cycle and cell division, including p53 and cdc25 (8). However, few have been reported concerning the correlation of Aurora kinases with cell cycle regulation and proliferation in ccRCC.

We aimed to explore the mechanisms by which Aurora kinases exerted its effects on ccRCC by downregulation the expression of the Aurora kinases with miRNAs and VX680 (small-molecule pan Aurora kinases inhibitor). We observed that silencing the expression of Aurora kinases induced the inhibition of the proliferation and metastasis, and lead to the 
G2/M phase arrest in ccRCC cells. Furthermore, we confirmed the anti-tumor activity of inhibition of Aurora kinases in HF assay and in a xenograft model. We observed that silencing Aurora kinases with miRNAs or treating the cells with VX680 could inhibit the phosphorylation of ERK, therefore decrease the expression of cdc25c, cyclinB/cdc2 and upregulate the expression and p-cdc2 (Tyr15). These changes led to inhibition of proliferation, metastasis and the G2/M arrest in ccRCC; moreover both Aurora kinases might be important targets for regulation of the tumor growth.

Therefore, inhibition of Aurora kinases might contribute to blocking the ccRCC progression. Aurora kinases appear to be potential therapeutic targets in the management of the renal cell carcinoma.

\section{Materials and methods}

Cell culture. Caki-1 and SN12C cells were kindly provided by B.T.T. (National Cancer Center, Singapore). The cells were maintained in DMEM medium (Invitrogen) supplemented with $10 \%$ fetal bovine serum (FBS; Invitrogen), $100 \mathrm{IU} / \mathrm{ml}$ penicillin and $100 \mu \mathrm{g} / \mathrm{ml}$ streptomycin (Invitrogen) in a humidified incubator containing $5 \% \mathrm{CO}_{2}$ at $37^{\circ} \mathrm{C}$.

Generation of the stable knockdown Aurora A and Aurora B in the SN12C and Caki-1 cell lines. SN12C and Caki-1 cells were seeded at a density of $1 \times 10^{5}$ cells per well in 6-well plates. Following overnight incubation, the cells reached 50\% confluence. They were then transfected with either the AurA miRNA, AurB miRNA or control miRNA vector (BLOCK-iT ${ }^{\mathrm{TM}} \mathrm{Pol}$ II miR RNAi Expression Vector kit with EmGFP, purchased from Invitrogen) using Lipofectamine 2000 (Invitrogen), according to the manufacturer's recommended protocol. In brief, the normal cellular medium was replaced with serum- and antibody-free DMEM medium. The miRNAs and Lipofectamine mixture were added directly to the six plates without removal of the culture medium. The medium was mixed by gentle agitation. The plates were incubated for an additional $6 \mathrm{~h}$ and then changed to normal medium. The initial selection for transfected cells was performed by growth in fresh medium containing $8 \mu \mathrm{g} / \mathrm{ml}$ Blasticidin S HCl (Invitrogen). Selective pressure was maintained by growth in a medium containing $8 \mu \mathrm{g} / \mathrm{ml}$ Blasticidin S HCl. After two weeks of growth in selective media, the clone with green fluorescence was selected and continually cultured in the normal medium. Cells were harvested for western blot analysis the expression of Aurora kinases. Stable transfected cells with AurA miRNA, AurB miRNA were designated Caki-1/AurA1, Caki-1/AurA2, Caki-1/AurB1, Caki-1/AurB2, SN12C/AurA, SN12C/AurB. Stable transfected cells with control miRNA were designated Caki-1/C, SN12C/C.

Analysis of cell proliferation and viability. Cells were seeded on 96-well plates in DMEM medium supplemented with $10 \%$ fetal bovine serum. Cells were treated with DMSO or VX680 for $96 \mathrm{~h}$ and then cell viability was measured with the Cell Counting Kit-8 (CCK-8; Dojindo Laboratories, Kumanmoto, Japan).

Cells (stably transfected with control miRNA, AurA miRNA, AurB miRNA, respectively) were seeded on 96-well plates in
DMEM medium supplemented with 10\% FBS and the proliferation of the cells was monitore by CCK- 8 assay at 24, 48, 72 and $96 \mathrm{~h}$.

Cell cycle analysis. Cells (stably transfected with control miRNA, AurA miRNA, AurB miRNA, respectively) were cultured in DMEM medium supplemented with $10 \%$ FBS for $96 \mathrm{~h}$ or cells were incubated with either VX680 or DMSO (control) for $96 \mathrm{~h}$. Those cells were then collected and analyzed using a cellular DNA flow cytometric analysis kit (Roche). Briefly, cells were collected after treatment and stained with propidium iodide. Cell cycle profiles were determined by flow cytometric analysis.

In vitro invasion assay. Invasion was determined using a variation of the Boyden chamber assay, as described (9). Briefly, cells were trypsinized and counted; next, $1 \times 10^{6}$ cells (SN12C cells treated with DMSO or $0.48 \mu \mathrm{mol} / 1 \mathrm{VX} 680\left(0.2 \mathrm{IC}_{50}\right)$; SN12C cells were stably transfected with control miRNA, AurA miRNA, AurB miRNA, respectively) suspended in $200 \mu \mathrm{l}$ of DMEM containing $0.1 \%$ BSA. The cells were seeded into the upper compartment (Costar) coated polycarbonate filter with a pore size of $8.0 \mu \mathrm{m}$ in a 24 -well plate. Each polycarbonate filter had been coated with $10 \mu \mathrm{l}$ of $0.5 \%$ Matrigel before the addition of cells. DMEM medium (600 $\mu \mathrm{l}$ ) containing 10\% FBS was added to the lower compartment as a chemo attractant. After $14 \mathrm{~h}$ of incubation at $37^{\circ} \mathrm{C}$ in $5 \% \mathrm{CO}_{2}, 90 \%$ relative humidity, the cells on the underside of the chamber were fixed to the membrane using methanol for $10 \mathrm{~min}$. Filters were stained with HE stain at room temperature. Cells in the upper compartment were removed using a cotton swab, leaving only the cells on the underside of the filter, representing those cells that had successfully invaded across the Matrigel-coated filter. The chambers were then photographed to compare the amount of invasive cells on the underside of the membrane. The five visual fields were photographed in every membranes, and manual counting of nuclear-stained cells. All samples were run in triplicate.

The hollow fibre assay (HF assay). The cells were harvested by a standard trypsinisation procedure and resuspended at the desired cell density $\left(1 \times 10^{6}\right.$ cells $\left./ \mathrm{ml}\right)$. The cell suspension was flushed into the hollow fibres, thereafter they were heat-sealed and cut at $1-\mathrm{cm}$ intervals. The fibres were incubated in DMEM medium in 6-well plates $24 \mathrm{~h}$ prior to surgical implantation in 6- to 8-week-old female pure strain Balb/c-nu/nu mice (Vital River Laboratory Animal Technology Co. Ltd). All animals were housed in controlled environment at $25^{\circ} \mathrm{C}$ on a $12 \mathrm{~h} \mathrm{light,}$ $12 \mathrm{~h}$ dark cycle. Mice were maintained in accordance with the National Institute of Health Guide for the Care and Use of Laboratory. Fibres were implanted s.c. in the back of the mice (two fibres per mouse, one for SN12C cells, one for Caki-1 cells). Separate in vitro control fibres were also prepared and were incubated in DMEM medium during the experiment (10 days). The mice were treated on day 3 with VX680 at $80 \mathrm{mg} / \mathrm{kg}$ by i.p. or by $50 \%$ PEG (6 mice per group). The mice were sacrificed at day 10 and the fibres were excised from the mice. Excess host tissue was removed and the cells were retrieved from the fibres (both in vitro and in vivo) for analysis of growth (the 'stable end-point' modified MTT assay) and cell cycle distribution (flow 
cytometric analysis to measure cell cycle distribution of the cell population), as described previously (10). For each measurement 10,000 cells were counted. The total number of cells in these fractions in cell cycle analysis was set at $100 \%$ (11).

Tumorigenicity of the cells with silencing Aurora kinases in the xenograft model. Athymic nude mice (Balb/c-nu/nu female, 6-8-week-old) were purchased from Vital River Laboratory Animal Technology Co. Ltd. Each cell line [SN12C cells stably transfected with control miRNA (SN12C/C), AurA miRNA (SN12C/AurA) and AurB miRNA (SN12C/AurB), respectively] was typsined, and washed twice with PBS. The $5 \times 10^{6}$ cells suspended in $200 \mu \mathrm{l} 0.9 \% \mathrm{NaCl}$ were injected s.c. into the left flank of Balb/c-nu/nu mice, five mice per group. Tumor dimensions were measured twice every week and the volume calculated as length $\mathrm{x}$ width $\mathrm{x}$ depth $\mathrm{x} 0.5$. Percentage of relative tumor volume calculated as the tumor volume on each day divided by the volume at the time of the first measurement. Mice were euthanized after the injection on the 22nd day. Tumors were removed, cleaned from adjacent tissues and weighed.

Tumor implantation and growth in a ccRCC xenograft model. Six-week-old female BALB/c-nu/nu nude mice (Vital River Laboratory Animal Technology Co. Ltd) had five million SN12C cells subcutaneously implanted in the left flank. When tumors had grown to an average volume of 100 to $150 \mathrm{~mm}^{3}$, tumor-bearing mice were separated into two groups of 7 animals. One group received i.p. injections of 50\% PEG300 as a vehicle control; one group received i.p. injections of VX680 at $80 \mathrm{mg} / \mathrm{kg}$ every day. Tumor size was measured 2-3 times per week and tumor volume was calculated as length $\mathrm{x}$ width $\mathrm{x}$ height $\mathrm{x} 0.5$. Percentage of relative tumor growth calculated as the tumor volume on each day divided by the volume at the time of the first measurement. The tumor growth ratio is presented as mean $\pm \mathrm{SD}$. Growth curves were plotted to show the mean relative tumor volume (RTV) within each experimental group at the indicated time points. Mice were euthanized at the end of the treatment period (on day 30). Tumors were removed, cleaned from adjacent tissues, weighed and fixed in $4 \%$ polyformaldehyde, paraffin-embedded, and then $4-\mu \mathrm{m}$-thick sections were prepared. All sections were stained with H\&E and were used for subsequent immunohistochemical analysis. Parts of all sections were stored at $-80^{\circ} \mathrm{C}$ for western blot analysis.

Cell lysate and western blot analysis. Lysates (cells treatment with VX680 or DMSO) were prepared by washing cells with PBS and then following the methods previously described (12). Portion of four randomly selected tumors from each group were homogenized for lysate preparation as previously described (12). For western blot analysis, samples transferred to a nitrocellulose membrane by semi-wet electrophoresis (Invitrogen) were incubated with primary antibody (rabbit anti-Aurora A, rabbit anti-Aurora B, rabbit anti-phosphorylated Aurora A (Thr288), mouse anti-phosphorylated Aurora B (Thr232), rabbit anticdc25c, rabbit anti-phosphorylated p-cdc2 (Tyr15), mouse anti-cdc2, mouse anti-cyclin B, rabbit anti-phosphorylated p-ERK, rabbit anti-ERK, rabbit anti-PCNA) overnight at $4^{\circ} \mathrm{C}$, detected with horseradish peroxidase-conjugated anti-rabbit or anti-mouse IgG (Santa Cruz), and developed using an
ECL Western blot detection and analysis system (Applygen Technologies Inc., Beijing, China). Membranes were tested for equal loading by probing for actin.

Immunohistochemistry. Immunohistochemical staining was done on $4-\mu \mathrm{m}$ formalin-fixed, paraffin-embedded tissue sections. Endogenous peroxidase activity was blocked with $3 \%$ hydrogen peroxide. Antigen retrieval was carried out in citrate buffer $(10 \mathrm{mmol} / \mathrm{l}, \mathrm{pH} 6.0)$ for $15 \mathrm{~min}$ at $100^{\circ} \mathrm{C}$ in a microwave oven. The slides were incubated with a primary rabbit rabbit anti-PCNA, overnight at $4^{\circ} \mathrm{C}$. Sections were then incubated with secondary anti-rabbit IgG (Santa Cruz) for $30 \mathrm{~min}$. After washing with $1 \mathrm{X}$ TTBS, sections were incubated with Vectastain ABC reagent (Santa Cruz). The immune complex was visualized using DAB substrate solution (Santa Cruz). For the quantitation of PCNA, see the description in Huang et al (13).

Statistical analysis. All values are expressed as mean \pm SD. Values were compared using Student's t-test. $\mathrm{P}<0.05$ was considered significant.

\section{Results}

Downregulation of Aurora kinases by miRNAs or VX680 induces the inhibition of the proliferation in SN12C and Caki-1 cells in vitro. To assess the activities of Aurora kinases in the growth of ccRCC cells, we employed the miRNAs targeting either Aurora A or B to knockdown the expression of Aurora kinases in both SN12C cells and Caki-1 cells. The basal and active level of the Aurora A or B was detected by western blot analysis (Fig. 1A). In both SN12C and Caki-1 cells, silencing of Aurora kinases caused significant reduction on basal and active levels of Aurora kinases A and B.

We then detected the potential effects of miRNA-mediated silencing of Aurora kinases on the growth of Caki-1 and SN12C cells in vitro by CCK-8 assay. Our results showed that cell growth significantly slowed down after silencing of Aurora kinases by miRNAs targeting Aurora A and Aurora B compared with that in control in both cell lines (Fig. 1B and C).

In order to confirm the active role of Aurora kinases on proliferation in ccRCC cell lines, we conducted the CCK-8 assay to detect the antiproliferation effect of a small-molecule pan Aurora kinases inhibitor, VX680, which has inhibition constants (Ki) of $0.6,18$ and $46 \mathrm{nM}$ for Aurora A, B and C, respectively (14). SN12C and Caki-1 cells were cultured in mediums with different concentrations of VX680 for $96 \mathrm{~h}$. It was found that VX680 obviously decreased the growth of cells in a dose-dependent manner (Fig. 1D) in both cell lines. Half inhibition concentration $\left(\mathrm{IC}_{50}\right)$ was $2.41 \pm 0.41 \mu \mathrm{mol} / \mathrm{l}$ and $0.478 \pm 0.11 \mu \mathrm{mol} / 1$ in SN12C and Caki-1 cells, respectively, the antiproliferation effect of VX680 was similar as that of silencing of Aurora kinases by miRNAs, indicating Aurora A and Aurora B exerted activity on proliferation in both SN12C and Caki-1 cells.

miRNAs targeting Aurora kinases or VX680 lead to the G2/M arrest in SN12C and Caki-1 cells in vitro. Since we had observed the antiproliferation effect induced by downregulation of Aurora kinases, we speculated that the antitumor activity was due to 
A

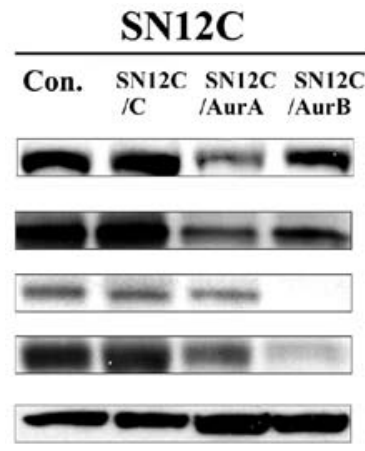

Caki-1

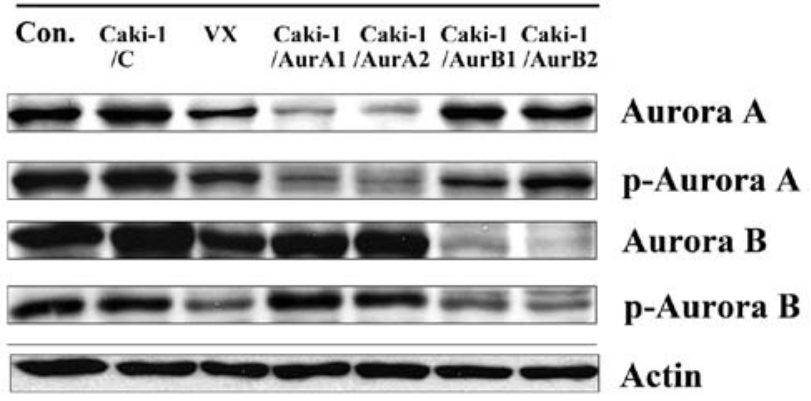

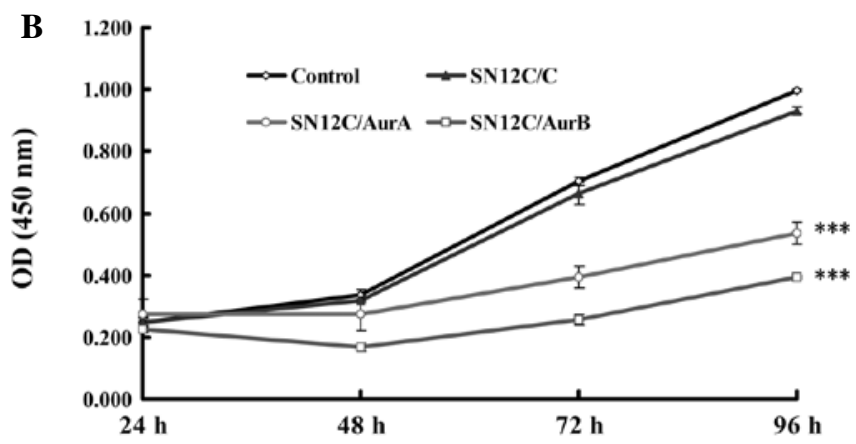

D

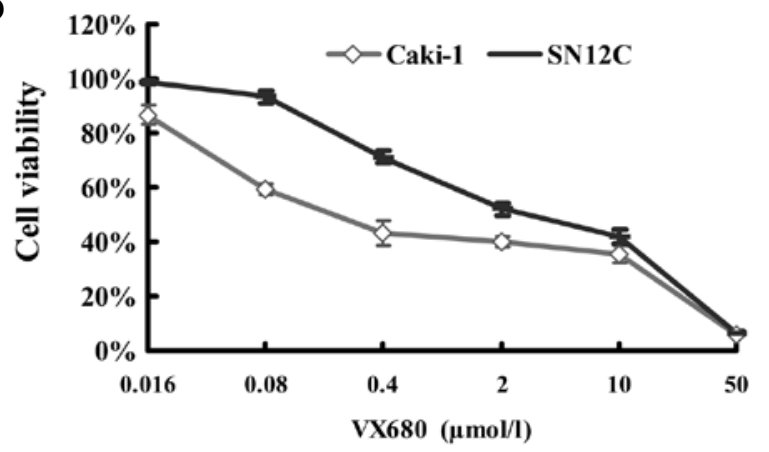

$\mathbf{E}$

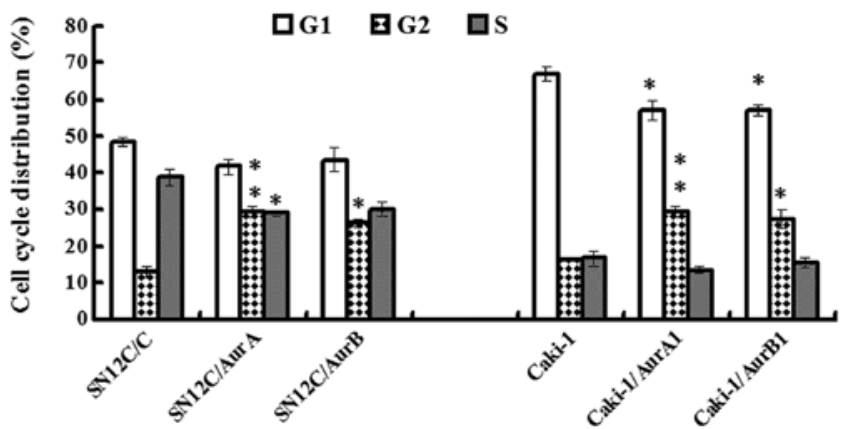

$\mathbf{F}$

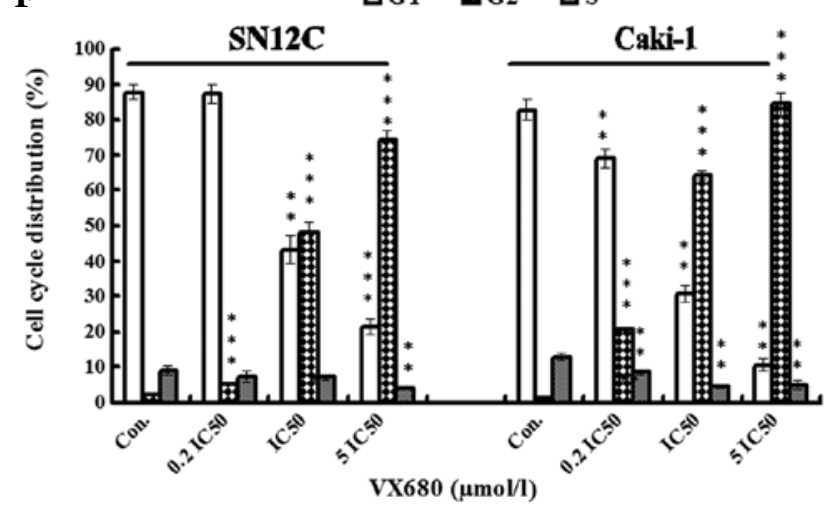

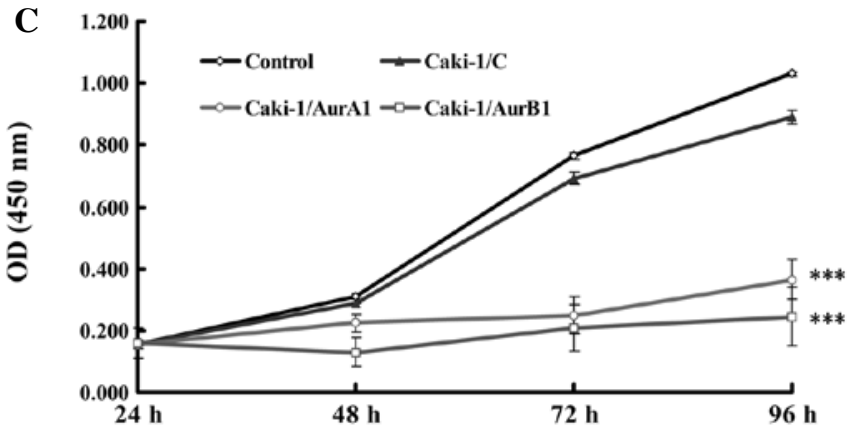

Figure 1. Antiproliferation and cell cycle arrest caused by inhibition of Aurora kinases. (A) The expression of Aurora A, Aurora B, p-Aurora A and p-Aurora B in whole-cell lysates in SN12C and Caki-1 cells silencing of Aurora A and Aurora B analyzed by western blot analysis. Con, untreated control samples; VX, samples treated with VX680 $0.1 \mu \mathrm{mol} / 1\left(\sim 0.2 \mathrm{IC}_{50}\right)$ for $96 \mathrm{~h}$. (B) The growth curve of proliferation of SN12C cells transfected with control miRNA (SN12C/C), AurA miRNA (SN12C/AurA) and AurB miRNA (SN12C/AurB). Error bars represented standard deviation. ${ }^{* * * *} \mathrm{P}<0.001$ indicates statistically significant divergence from the non-target control cells (SN12C/C). (C) The growth curve of proliferation of Caki-1 cells transfected with control miRNA (Caki-1/C), AurA miRNA (Caki-1/AurA1) and AurB miRNA (Caki-1/AurB1). Error bars represented standard deviation. ${ }^{* * *} \mathrm{P}<0.001$ indicates statistically significant divergence from the non-target control cells (Caki-1/C). (D) Effect of VX680 on the viability of SN12C and Caki-1 cell lines. Cells were treated with VX680 for $96 \mathrm{~h}$ and cell viability was determined by a CCK-8 assay. Error bars represented standard deviation. (E) miRNAs mediated Aurora kinase silencing-induced cell cycle arrest in G2/M. SN12C and Caki-1 cells transfected with control miRNA, AurA miRNA and AurB miRNA were cultured for $96 \mathrm{~h}$, stained with Annexin V-FITC and propidium iodide (PI) and analyzed by flow cytometry. Error bars represent standard deviation. ${ }^{*} \mathrm{P}<0.05,{ }^{* *} \mathrm{P}<0.01$ indicates statistically significant divergence from the non-target control cells. (F) VX680 induced cell cycle arrest in G2/M. SN12C and Caki-1 cells were incubated with VX680 for 96 h, stained with Annexin V-FITC and PI and analyzed by flow cytometry. Error bars represent standard deviation. ${ }^{* *} \mathrm{P}<0.01$, ${ }^{* * *} \mathrm{P}<0.001$ indicates statistically significant divergence from the control cells.

the cell cycle arrest. Several reports have showed that Aurora kinases are critically involved in cell cycle regulation and hence in proliferation in some types of tumors (15). Therefore, we tested whether deregulation of Aurora kinases induced by knocking down Aurora kinases or VX680 might have effect on the cell cycle profile in SN12C and Caki-1 cells. We found that inhibition of either Aurora A or B by miRNAs also induced cell accumulation in G2/M phase in both of the two cell lines (Fig. 1E), whereas a decrease in G1 phase in Caki-1 cells. When the cells were treated with increased concentrations of VX680 for $96 \mathrm{~h}$, we observed that VX680 led to significant G2/M arrest and reduction of G1 (Fig. 1F) in a dose-dependent manner. Cells accumulated almost completely in $\mathrm{G} 2 / \mathrm{M}$ in both cells treated with $5 \mathrm{IC}_{50}$ VX680 (2.4 and $12.0 \mu \mathrm{mol} / 1$ for Caki-1 and SN12C 
A

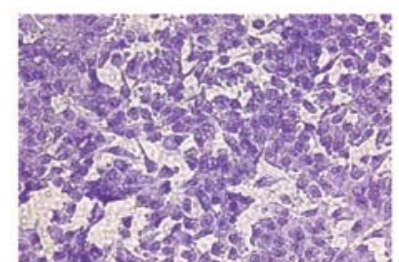

$\mathrm{SN} 12 \mathrm{C} / \mathrm{C}$

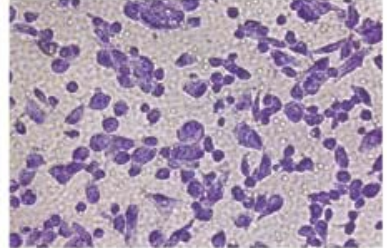

SN12C/AurA

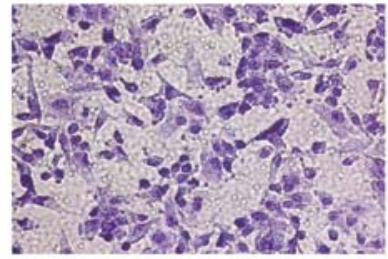

SN12C/AurB
B

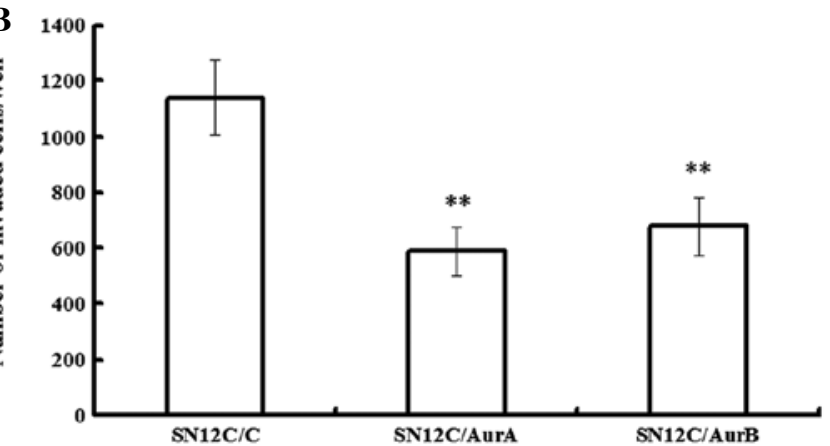

D

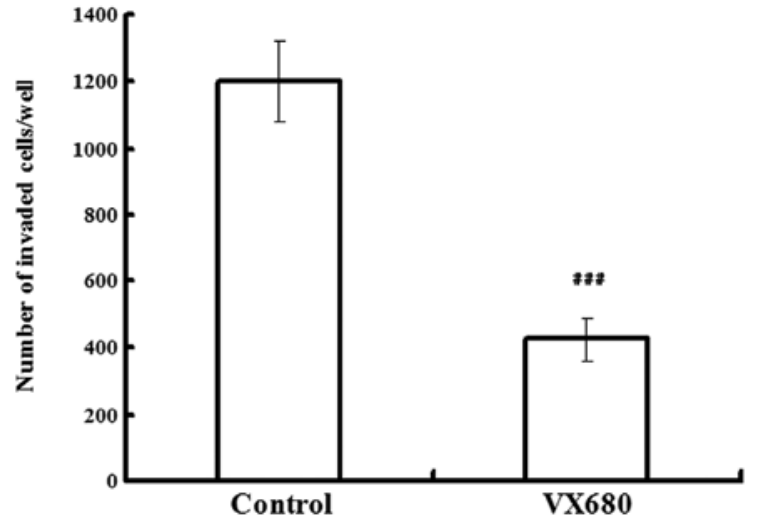

cells, respectively) for $96 \mathrm{~h}$. These results were consistent with the prediction that the Aurora kinases were crucial for cell cycle progression in ccRCC, suggesting that inhibition of Aurora kinases could arrest the ccRCC cells in G2/M phase and the effect of Aurora kinases on proliferation was partly due to the activity on the cell cycle in ccRCC cells.

miRNAs targeting Aurora kinases or VX680 inhibit the metastasis in SN12C cells in vitro. In order to determine whether Aurora kinases were involved in mediating the invasiveness of ccRCC cells, we conducted transwell assays to examine the role of Aurora kinases in invasion of SN12C cell lines. Equal numbers of cells $\left(1 \times 10^{6} / \mathrm{ml}\right)$ by treatment of either silencing of Aurora kinases or being treated with either DMSO or $0.48 \mu \mathrm{mol} / 1 \mathrm{VX} 680\left(0.2 \mathrm{IC}_{50}\right)$ were allowed to invade through a membrane coated with Matrigel, toward a chemo attractant (10\% FBS) for $14 \mathrm{~h}$. The invaded cells were fixed, stained and counted. In SN12C cells, invasion was decreased by 48.30 and $40.58 \%$ following Aurora A and Aurora B depletion, while the vector control cells invaded in similar numbers to the parental SN12C cells (Fig. 2A and $\mathrm{B}$ ), indicating that the Aurora A and B knockdown cells were considerably less invasive than the vector control cells. VX680 treated cells exhibited a decrease in invasion of up to $64.59 \%$, compared with SN12C control (Fig. 2C and D). Our
C

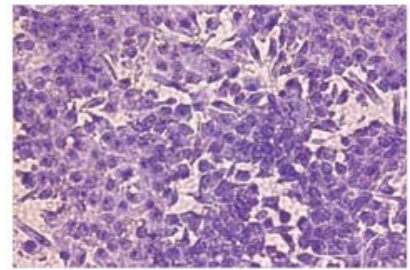

Control

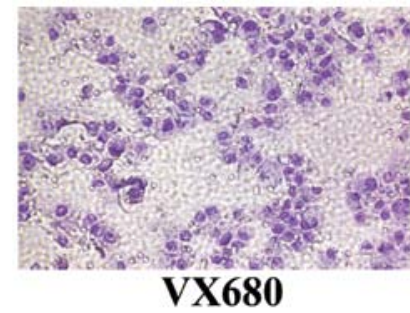

VX680
Figure 2. The effect of Aurora kinases silencing or VX680 on invasion in SN12C cells in vitro. (A and B) Equal numbers of SN12C cells transfected with control miRNA (SN12C/C), AurA miRNA (SN12C/AurA) and AurB miRNA (SN12C/AurB) were seeded into the upper compartment of a transwell system and allowed to invade through Matrigel substrate for $14 \mathrm{~h}$. The invaded cells were then fixed and counted. Number of invaded cells were less in SN12C/AurA and SN12C/AurB than in SN12C/C cells; error bars represent the standard deviation and an asterisk indicates statistically significant divergence from the non-target control clones; ${ }^{* *} \mathrm{P}<0.01$. (C and D) The effect was observed in the VX680 treatment cells, which exhibited a decrease in invasion of $64.59 \%$ compared with SN12C cells. Error bars represent the standard deviation and

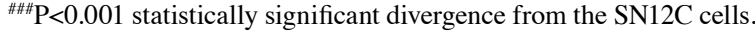

data showed that downregulation of Aurora kinases inhibited the metastasis in SN12C cells.

Inhibition of Aurora kinases by VX680 induces growth inhibition and cell cycle arrest in SN12C and Caki-1 cells tested by $H F$ assay. We used HF assay to detect the antitumor effect of inhibition of Aurora kinases by VX680. The hollow fibres were quite well tolerated by the nude mice. The VX680 treatment did not affect the conditions of the mice beyond acceptable limits. From all experimental groups cell suspensions were retrieved from fibres of different animals ( 3 mice per group) to be assayed for cytotoxicity of VX680. Compared to the in vivo control fibres, VX680 treatment produced a significant reduction in growth of about 32.7 and $42.6 \%$ in SN12C and Caki-1 cells, respectively $(\mathrm{P}<0.01)$ (Fig. 3A). Although the cell growth of in vivo control fibres was a little slower than in in vitro control fibre, there were no significantly difference in two groups ( $\mathrm{P}>0.05)$ (Fig. 3A).

From all experimental groups cell suspensions were retrieved from fibres of different animals (3 mice per group) to be assayed for cell cycle phase distribution (Fig. 3B). The tendency for increased G2/M-phase in vivo control fibres was found in SN12C and Caki-1 cells compared with the fibres from the in vitro control. In Caki-1 cells, G2/M phase in the in vivo control fibres was significant different from that in the in vitro 

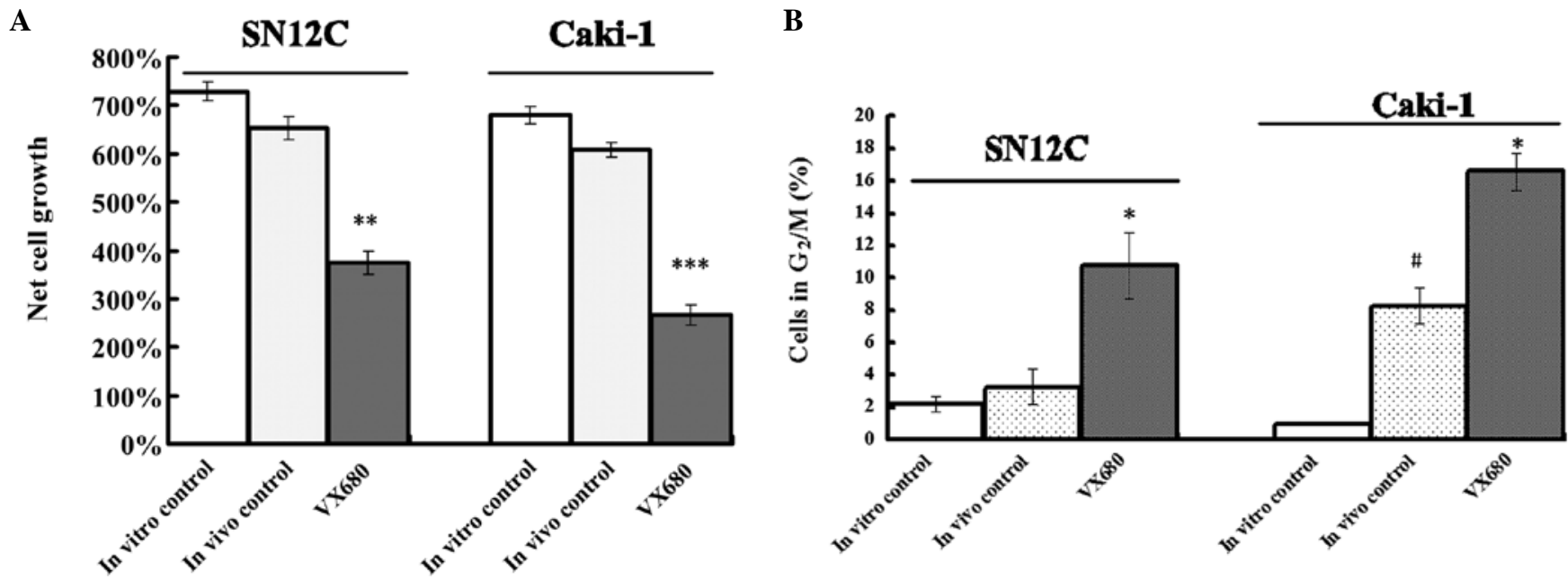

Figure 3. The growth inhibition and cell cycle arrest of VX680 in SN12C and Caki-1 cells tested by HF assay. (A) Net cell growth (\%) of SN12C and Caki-1 cells grown in hollow fibers implanted in nude mice. Tumor cells were seeded at $1 \times 10^{6}$ cells $/ \mathrm{ml}$ in hollow fibers and incubated overnight. Fibers were implanted into mice at s.c. sites. The mice were treated with VX680 or 50\% PEG300. In vitro control: fibres (per cell line three fibres) were incubated in DMEM culture medium. At 10 days mice were sacrificed, fibers excised and the MTT assay performed to determine cell viability. Results are expressed as mean $\%$ net cell growth. Error bars represented the standard deviation and an asterisk indicated statistically significant divergence to in vivo fibre control, ${ }^{* *} \mathrm{P}<0.01,{ }^{* * *} \mathrm{P}<0.001$. (B) Cell cycle distribution for SN12C and Caki-1 cells after retrieval from the hollow fibres. Per cell line three fibres from different mice were assayed using flow cytometry analysis. Total viable cell population was set at $100 \%$. In vitro control: fibres were incubated in DMEM culture medium. Error bars represented the standard deviation and an asterisk indicates statistically significant divergence to in vivo fibre control: ${ }^{*} \mathrm{P}<0.05 ;{ }^{*} \mathrm{P}<0.05$ indicates statistically significant divergence to in vitro fibre control.

Table I. Effects of miRNA-mediated Aurora kinase silencing on SN12C tumor growth in athymic mice.

\begin{tabular}{|c|c|c|c|c|c|c|}
\hline \multirow[b]{2}{*}{ Group } & \multirow[b]{2}{*}{ No. of animals (n) } & \multicolumn{2}{|c|}{ Body weight (g) } & \multicolumn{3}{|c|}{ Tumor size } \\
\hline & & Start & End & Volume $\left(\mathrm{mm}^{3}\right)$ & RTV & $\mathrm{T} / \mathrm{C}(\%)$ \\
\hline $\mathrm{SN} 12 \mathrm{C} / \mathrm{C}$ & $5 / 5$ & $17.94 \pm 0.50$ & $19.57 \pm 0.77$ & $2,042.04 \pm 542.80$ & $23.86 \pm 4.44$ & \\
\hline SN12C/Aur A & $5 / 5$ & $17.84 \pm 0.84$ & $19.58 \pm 0.72$ & $802.68 \pm 350.27^{* * *}$ & $8.50 \pm 2.69^{* * *}$ & 35.62 \\
\hline SN12C/Aur B & $5 / 5$ & $17.45 \pm 0.69$ & $19.06 \pm 1.19$ & $1,289.59 \pm 290.13^{* *}$ & $13.71 \pm 1.92^{* *}$ & 57.44 \\
\hline
\end{tabular}

${ }^{*} \mathrm{P}<0.05 ;{ }^{* *} \mathrm{P}<0.01 ;{ }^{* * *} \mathrm{P}<0.001$ vs. control miRNA; $\mathrm{T} / \mathrm{C}(\%), \mathrm{T}_{\mathrm{RTV}} / \mathrm{C}_{\mathrm{RTV}} \times 100 ; \mathrm{RTV}$, relative tumor volume.

control fibres. In VX680-treated mice, VX680 treatment resulted in a clear $\mathrm{G} 2 / \mathrm{M}$-phase arrest for both cell lines $(\mathrm{P}<0.05)$. The effect of VX680 on cell cycle distribution observed in HF assay was similar as the activity of VX680 in vitro experiments.

Downregulation of Aurora kinases by silencing Aurora kinases or VX680 inhibits the growth in SN12C xenografts. As described above, the effect of inhibition of Aurora kinases A and B on growth of ccRCC was partly identified in vitro and semi in vivo experiments, next we investigated it in vivo. We explored whether the downregulation of Aurora kinase expression in SN12C cells would affect their ability to develop tumors in nude mice. We injected subcutaneously SN12C cells (SN12C/C, SN12C/AurA, $\mathrm{SN12C/AurB}$ ) into nude mice (five nude mice per cell line) and observed the growth of tumors. The tumors of SN12C transfected with AurA miRNA and AurB miRNA grew slower than that in vector control (Fig. 4A). The growth rate was 35.62 and $57.44 \%$ comparing to control, respectively, according to RTV (Table I), indicating that silencing of Aurora kinases slowed down the growth of SN12C cells in xenografts.
To further investigate the role of Aurora kinases in growth of ccRCC in vivo, we evaluated the effect of inhibition of Aurora kinases by VX680 on tumor growth in an established SN12C xenograft model. As we had proved the effect of VX680 on Caki-1 xenografts (12), tumors in the control animals showed increased volumes and exponential growth (Fig. 4B), the group administrated with VX680 (80 mg/kg) suppressed tumor growth and reduced tumor volume compared with the control with $49.9 \%(\mathrm{P}<0.001)$ of $\mathrm{T} / \mathrm{C}$ ratio at the end of the treatment according to RTV and a $47.9 \%(\mathrm{P}<0.001)$ decrease according to tumor weight in SN12C xenografts (Fig. 4B and C, Table II). Treatment with VX680 did not alter animal body weight (Fig. 4D), peripheral blood counts, or other biological parameters (data not shown). These results implied that the antitumor effect of VX680-mediated Aurora kinase inhibition on the xenograft model was not due to systemic toxicity.

Inhibition of Aurora kinases by miRNAs or VX680 influences the expression of the cell cycle regulator in SN12C and Caki-1 cells in vitro. In order to find out whether the 
A

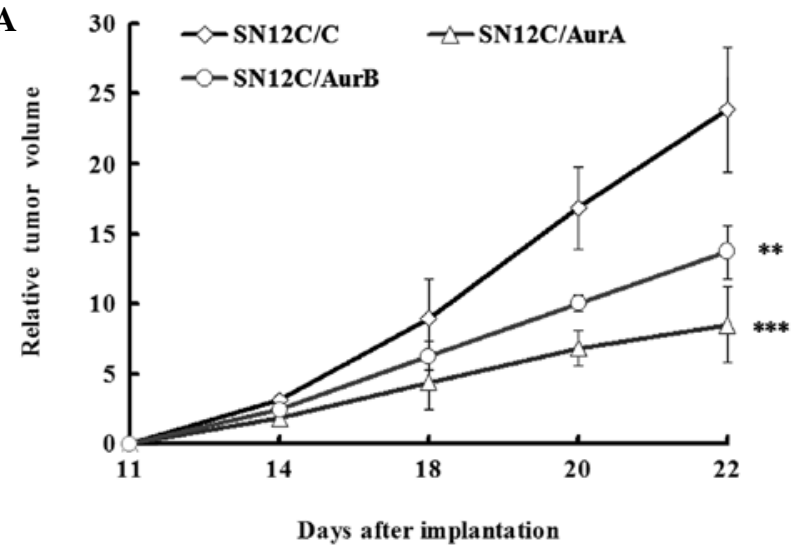

C

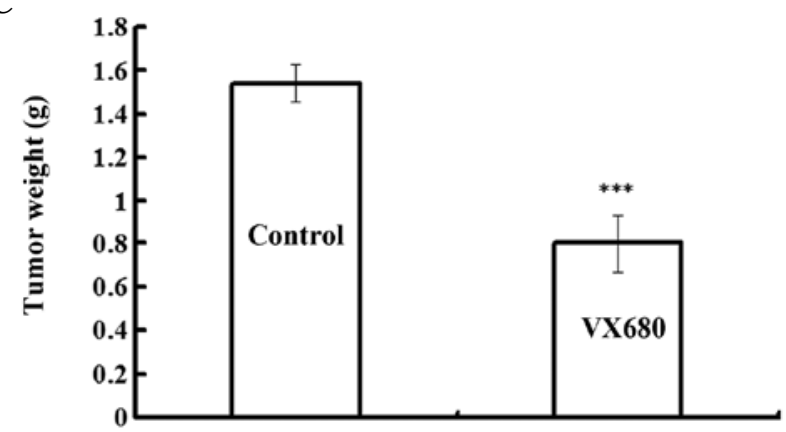

B

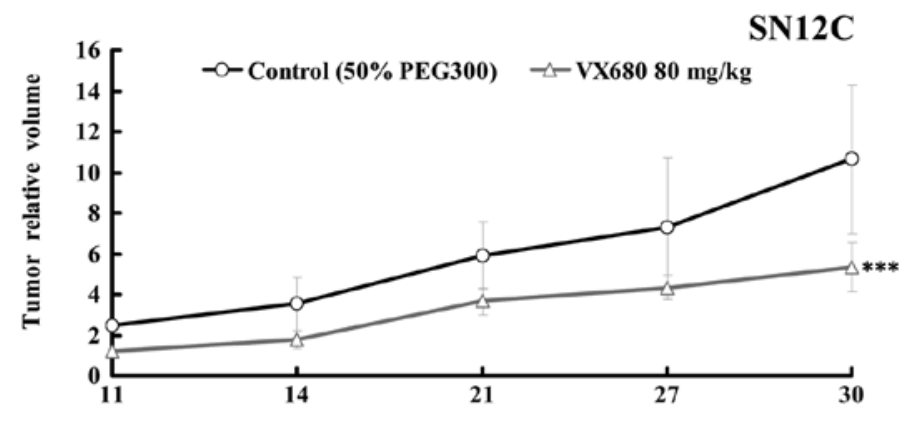

Days after tumor implantation

$\mathbf{D}$

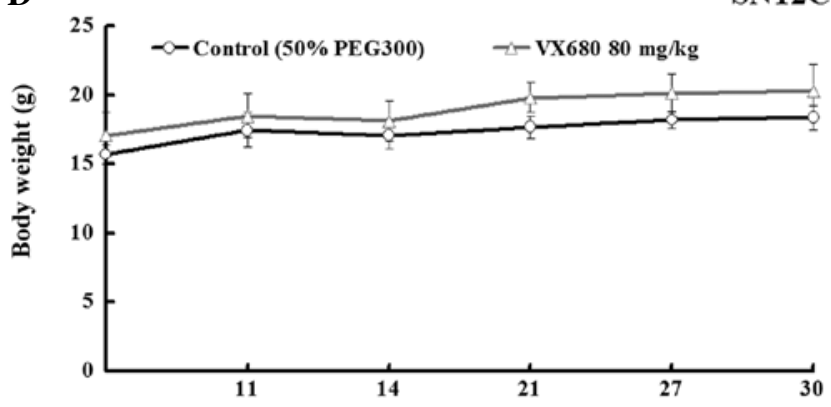

Days after tumor implantation

Figure 4. The effect of inhibition of Aurora kinases mediated by miRNA or VX680 on the growth in SN12C xenografts. (A) Changes in volume of SN12C/C, SN12C/AurA and SN12C/AurB (transfected with control miRNA, AurA miRNA and AurB miRNA respectively) xenograft tumors in mice. Cells were injected subcutaneously into female nude mice. Each point represents the mean of independent relative tumor volumes. Percentage of relative tumor volume calculated as the tumor volume on each day divided by the volume at the time of the first measurement. The growth of SN12C/AurA and SN12C/AurB was much slower than SN12C/C xenograft tumors ( $\left.{ }^{* *} \mathrm{P}<0.01,{ }^{* * *} \mathrm{P}<0.001\right)$. (B) Five million SN12C cells were subcutaneously injected into the left flank of each mouse to initiate ectopic tumors. Intraperitoneal injection of 50\% PEG300 or VX680 $(80 \mathrm{mg} / \mathrm{kg})$ started on day 7 after tumor cell inoculation and continued daily to day 30 . Xenograft tumor volumes $\left(\mathrm{mm}^{3}\right)$ were measured. Tumor growth curves (according to RTV) were plotted for SN12C xenografts with intraperitoneal injection of $80 \mathrm{mg} / \mathrm{kg}$ VX680 or $50 \%$ PEG300 ( $\mathrm{n}=7$ mice per group) at given time-points. Error bars represent standard deviation. ${ }^{* * * *} \mathrm{P}<0.001$ indicates statistically significant divergence from the control group. (C) The corresponding tumor weight at the endpoint for each group. ${ }^{* * *} \mathrm{P}<0.001$ indicates statistically significant divergence from the control group. (D) The effect of VX680 on body weight of nude mice carrying SN12C xenograft tumors. No effect on weight was observed after VX680 treatment. Error bars represented standard deviations.

Table II. Effects of VX680 on SN12C tumors in athymic mice.

\begin{tabular}{|c|c|c|c|c|c|c|c|c|c|}
\hline \multirow[b]{2}{*}{ Group } & \multirow[b]{2}{*}{$\begin{array}{c}\text { Dose } \\
(\mathrm{mg} / \mathrm{kg})\end{array}$} & \multirow[b]{2}{*}{$\begin{array}{c}\text { No. of } \\
\text { animals (n) }\end{array}$} & \multicolumn{2}{|c|}{ Body weight (g) } & \multicolumn{3}{|c|}{ Tumor size } & \multicolumn{2}{|c|}{ Tumor weight } \\
\hline & & & Start & End & $\begin{array}{l}\text { Volume } \\
\left(\mathrm{mm}^{3}\right)\end{array}$ & RTV & $\begin{array}{l}\mathrm{T} / \mathrm{C} \\
(\%)\end{array}$ & $(\mathrm{g})$ & $\begin{array}{c}\text { Inhibition } \\
(\%)\end{array}$ \\
\hline Control & & $7 / 7$ & $16.70 \pm 0.67$ & $19.36 \pm 0.93$ & $1,405.70 \pm 230.28$ & $10.65 \pm 3.01$ & & $1.54 \pm 0.09$ & \\
\hline VX680 & $80 \times 23$ & $7 / 7$ & $17.12 \pm 1.62$ & $20.28 \pm 1.90$ & $685.09 \pm 188.66^{* * * *}$ & $5.34 \pm 1.04^{* * * *}$ & 49.9 & $0.8 \pm 0.13^{* * *}$ & 47.9 \\
\hline
\end{tabular}

${ }^{*} \mathrm{P}<0.05 ;{ }^{* *} \mathrm{P}<0.01 ;{ }^{* * *} \mathrm{P}<0.001$ vs. control; $\mathrm{T} / \mathrm{C}(\%), \mathrm{T}_{\mathrm{RTV}} / \mathrm{C}_{\mathrm{RTV}} \times 100 ; \mathrm{RTV}$, relative tumor volume.

growth inhibition by VX680 was caused through inhibition of the activity of Aurora kinases in VX680-treated ccRCC cells, we examined basal and active level of Aurora kinases in the VX680 treated SN12C and Caki-1 cell lines by western blot. The results showed that VX680 potently inhibited the phosphorylation of Aurora A and Aurora B in both cell lines (Fig. 5A). Therefore, the antiproliferation activity of VX680 was mainly due to the inhibition of p-Aurora A and
p-Aurora B in SN12C and Caki-1 cells which was consistent with our previous results (12).

We further demonstrated that exposure of cells to VX680 changed the total amounts of Aurora A or Aurora B in mitosis to some extent, suggesting that the decreased phosphorylation of Aurora A and Aurora B was not due only to inhibition of phosphorylation, but also partly to Aurora kinase degradation or downregulation. 
A

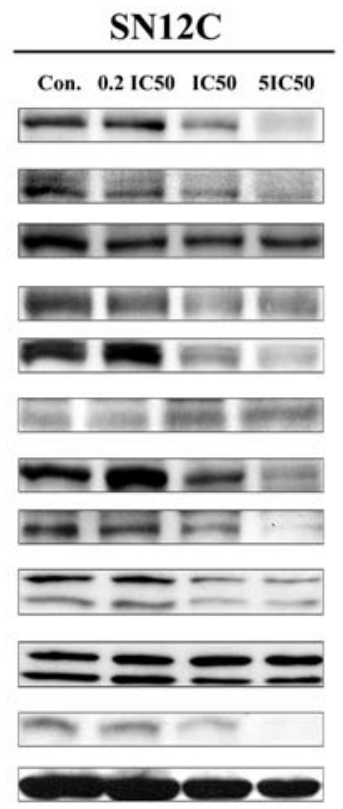

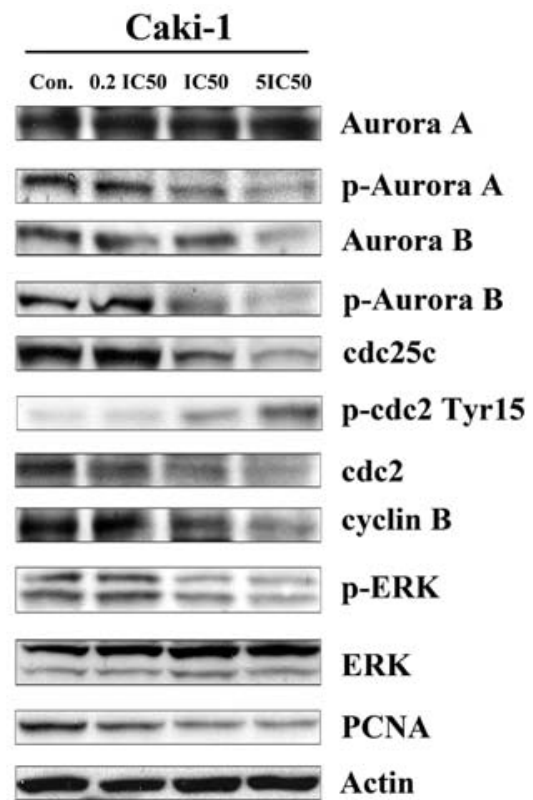

B

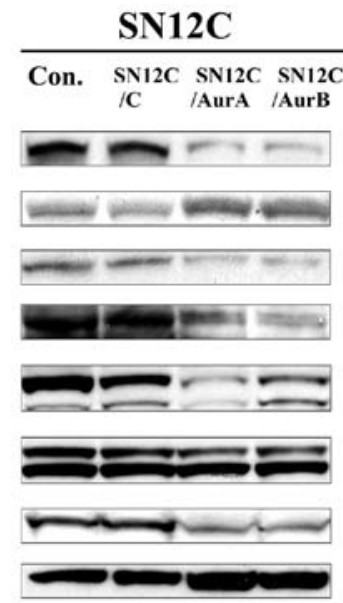

Caki-1

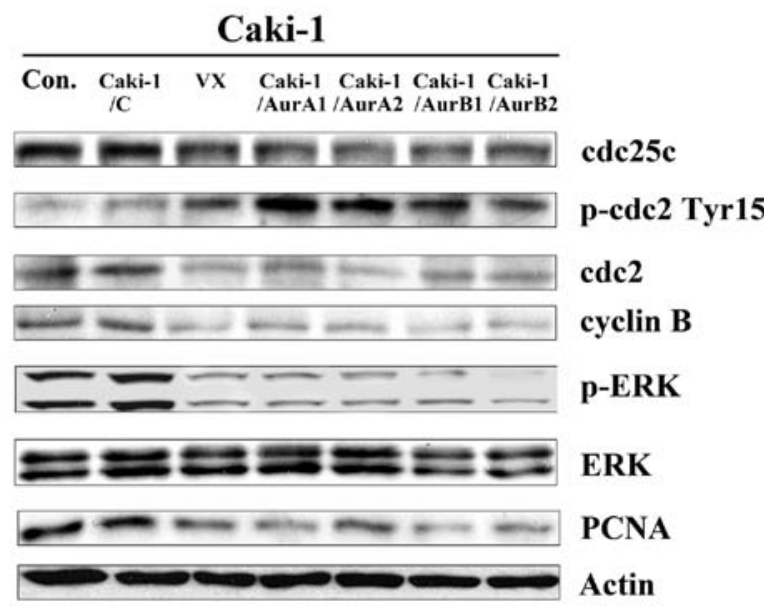

Figure 5. The effect of inhibition of Aurora kinases on cell cycle regulators in SN12C and Caki-1 cells in vitro. (A) SN12C and Caki-1 cells were incubated with increasing concentrations of VX680 for $96 \mathrm{~h}$. Whole-cell lysates were subjected to western blot analysis. (B) Lysates were prepared from SN12C, SN12C/C, SN12C/ AurA and SN12C/AurB cells, Caki-1, Caki-1/C, Caki-1/AurA1, Caki-1/AurA2, Caki-1/AurB1, Caki-1/AurB2 cells and Caki-1 cells treated with VX680 $0.1 \mu$ mol/1 $\left(\sim 0.2 \mathrm{IC}_{50}\right)$ for $96 \mathrm{~h}$. Con, untreated control samples; VX, samples treated with VX680, whole-cell lysates were subjected to western blot analysis. For western blot analysis, samples were transferred to a nitrocellulose membrane by semi-wet electrophoresis and incubated with indicated primary antibody (p-Aurora A, Aurora A, p-Aurora B, Aurora B, cdc25c, p-cdc2 (Tyr15), cdc2, cyclin B, PCNA, p-ERK1/2 (Thr202/Tyr204), ERK1/2). Actin was used as loading and transfer control. The experiment was repeated twice and similar results were obtained.

The literature shows that Aurora kinases are critically involved in cell cycle and thus in proliferation (15) in some tumors. Based on the effect of Aurora kinases on the cell cycle distribution proved by our experiments, we next conducted experiments to examine the mechanism of cell cycle arrest induced by inhibition of Aurora kinases, to observe the changes of cell cycle regulator in $\mathrm{SN} 12 \mathrm{C}$ cells and Caki-1 cells treated with AurA miRNA, AurB miRNA and VX680.

As reported, the cyclin $\mathrm{B} / \mathrm{cdc} 2$ complex (maturation/mitosis promoting factor) is thought to regulate progression through the $\mathrm{G} 2 / \mathrm{M}$ phase of the cell cycle (16). In our study, expression of cyclin B and cdc2 was significantly and dose-dependently decreased in treating cells with VX680 (Fig. 5A). Knockdown of Aurora $A$ and $B$ induced the downregulation of cyclin $B$ and cdc2 (Fig. 5B). Therefore, we draw the conclusion that Aurora kinases contributed to the regulation of cyclin $\mathrm{B} / \mathrm{cdc} 2$ in SN12C and Caki-1 cells.

The activity of cdc2 is negatively regulated by the phosphorylation of the amino acid residue threonine-14 (Thr14) and tyrosine-15 (Tyr15). Cdc25C, a phosphatase to remove the phosphate group from p-cdc2 (Tyr15), is the key component controlling the entry of cells into mitosis $(17,18)$. Therefore, we sought to determine the phosphorylation states of cdc2 and the expression of cdc25C in cells after inhibition of Aurora kinases. In this study, VX680 treatment resulted in a decrease in total $\operatorname{cdc} 25 \mathrm{c}$ and an increase in cdc2 phosphorylation at Tyr15 site in SN12C and Caki-1 cells (Fig. 5A). Consistent with these results, the increased expression of p-cdc2 (Tyr15) and decreased expression of cdc $25 \mathrm{c}$ was found in the cell silencing of Aurora kinases (Fig. 5B). 
A

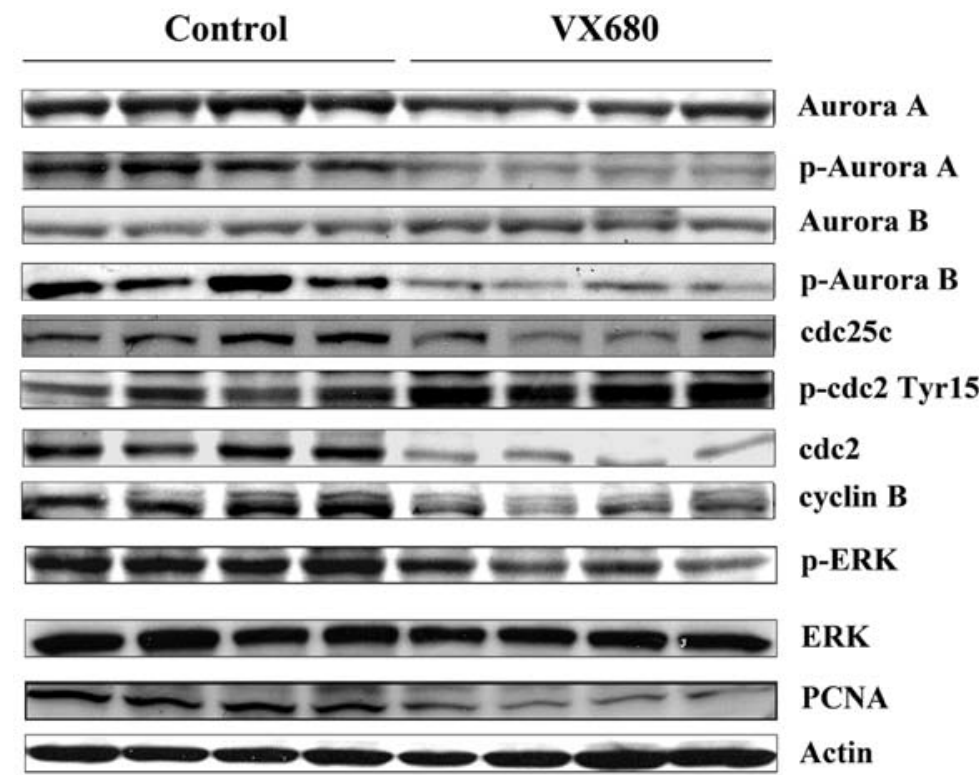

B

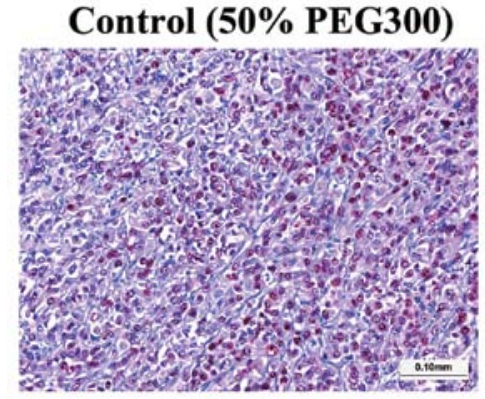

\section{C}

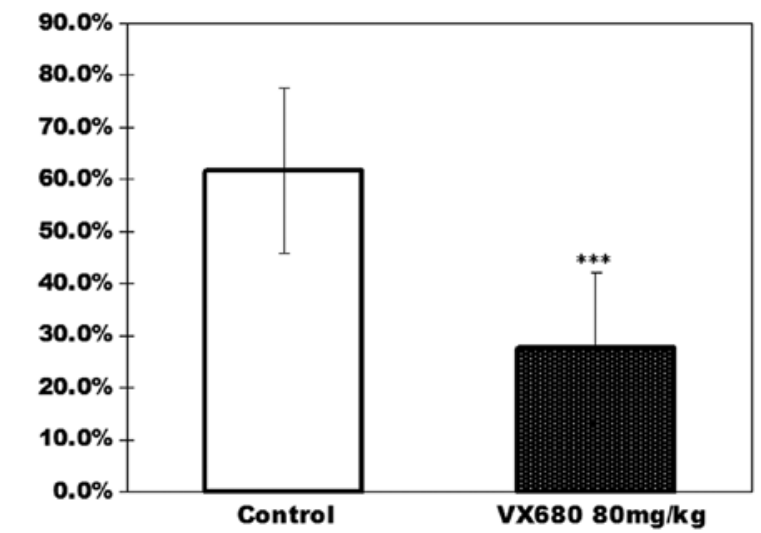

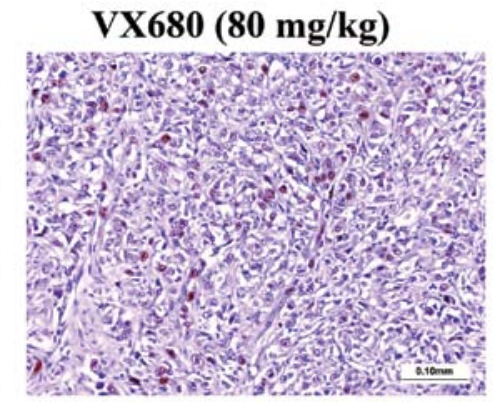

Figure 6. The effect of inhibition of Aurora kinases by VX680 on cell cycle regulators in SN12C xenografts. (A) A portion of four randomly selected tumors from each group (SN12C xenograft-bearing mice treated daily with VX680 or 50\% PEG300) were homogenized for lysate preparation and analyzed by western blot for protein expression. For western blot analysis, samples were transferred to a nitrocellulose membrane by semi-wet electrophoresis and incubated with primary antibody. The expression of $\mathrm{p}$-Aurora $\mathrm{A}$, Aurora A, p-Aurora B, Aurora B, cdc25c, p-cdc2 (Tyr15), cdc2, cyclinB, PCNA, p-ERK1/2 (Thr202/Tyr204), ERK1/2. Actin was used as loading and transfer control. The experiment was repeated twice and similar results were obtained. (B) Immunohistochemical staining for PCNA in SN12C xenograft tumors after treatment with VX680 or 50\% PEG300. (C) Quantification of a proliferation index (PCNA) staining in SN12C xenograft tumors. Error bars represent standard deviation. ${ }^{* * *} \mathrm{P}<0.0001$ indicates statistically significant divergence from the control group. Inhibition of Aurora kinases by VX680 decreased the expression of PCNA in SN12C xenograft tumors.
Members of the MAP kinase (MAPK) family have long been known to play an important role in regulating cell growth, differentiation, and cell death (19). One of them, the ERK pathway, is generally considered as a survival promotion pathway (20). To investigate whether Aurora kinases had an effect on the ERK pathway, the SN12C and Caki-1 cells were downregulated by the expression of Aurora kinase miRNAs or VX680, and then the phosphorylation status and basal levels of ERK was determined by western blot with specific antibodies. As shown in Fig. 5A and B, the phosphorylation of ERK was reduced in both cells when treated with Aur miRNA or VX680, but there was no influence on the expression of total ERK.

As we proved that downregulation of Aurora kinases inhibited the growth of SN12C and Caki-1 cells, we evaluated the level of PCNA (the proliferation marker), as shown in Fig. 5A, VX680 decreased the expression of PCNA in a dose-dependent fashion as determined by western blot analysis. The low level of PCNA was also observed in cell silencing of the expression of Aurora kinases (Fig. 5B).

Downregulation of Aurora kinases by VX680 affects the cell cycle regulator in SN12C xenografts. Tumor tissues obtained from mice on day 30 after tumor implantation were examined for expression and activity of Aurora kinases by western blot analysis. We found that relatively lower levels of p-Aurora A and p-Aurora B were detected in VX680-treated tumors when compared with the control tumors (Fig. 6A). These results were consistent with the results from the in vitro experiments, which 
showed a decrease of phosphorylation of Aurora kinases in the VX680-treated cells (Fig. 5A). We also found that cell proliferation within tumors, as indicated by PCNA, was markedly reduced, as analyzed by western blot analysis (Fig. 6A) and supported by PCNA immunohistochemical analysis. PCNA staining in control tumors showed $61.73 \pm 15.98 \%$ PCNApositive cells (proliferation index), whereas only $27.64 \pm 15.54 \%$ PCNA-positive cells were observed in the VX680-treated group, a decrease of $55.2 \%(\mathrm{P}<0.001)$ (Fig. 6B and $\mathrm{C}$ ).

Next we analyzed the alterations of cyclin $\mathrm{B} / \mathrm{cdc} 2$ expression in xenograft tumors after VX680 treatment in SN12C xenografts. We observed that cyclin B, cdc2 and cdc25c were downregulated in the VX680-treated group relative to the control group (Fig. 6A). Furthermore, the protein levels of p-cdc2 obviously increased in VX680-treated mice (Fig. 6A), whereas the active level ERK was decreased, the basal level of ERK did not change. The results were consistent with the results of the in vitro experiments, further confirming that the effect of inhibition of Aurora kinases on cell cycle regulator in ccRCC.

\section{Discussion}

Aurora A/B/C have been proved to be related with cancer progression and to be involved in the regulation of the cell cycle. They have been suggested as possible new anticancer targets (21). Despite previous studies of Aurora kinases on various tumors (22), the important roles of Aurora kinases and their signaling pathway in ccRCC is not fully elucidated. Our current study was an attempt to address this issue and to clarify the potent function of Aurora kinases in human ccRCC (23).

The functions of Aurora kinases were investigated through dowregulating the expression of Aurora kinases in the SN12C and Caki-1 lines. VX680 treatment or Aurora kinases silencing using miRNAs caused inhibition of the basal and active levels of Aurora kinases, following decrease in cell proliferation ability, leading to arrest in G2/M phase and inhibition of metastasis in both cell types. In HF assay, we found that the growth of SN12C and Caki-1 was inhibited and cells were arrested in $\mathrm{G} 2 / \mathrm{M}$ phases after treatment with VX680. Moreover, tumors injected SN12C cells with low expression of Aurora kinases induced by miRNAs grew more slowly than the tumors injected with SN12C control miRNA. In SN12C xenograft model, VX680 reduced the tumor growth through blocking the activity of Aurora kinases. Above results implied that Aurora kinases had some effects on proliferation in ccRCC.

The mechanism of cell cycle regulation is complicated and the cell cycle progression is tightly regulated by the synthesis/degradation, association/dissociation, translocation between cytoplasm and nucleius, and the phosphorylation/dephosphorylation of several protein regulators (24). The progression of cell from G2 to M phase requires the coordination of various regulatory proteins, and is initiated by formation of the cyclinB/cdc complex through the activation of cdc25c, leading cells into mitosis (25). Before entry to the mitotic phase, cdc2 is kept inactive by tyrosine-15 (Tyr15) and threonine-14 (Thr14) and dephosphorylation of the two inhibitory subunits Tyr15 and Thr14 by cdc $25 \mathrm{c}$ dual phosphatase will drive cells into mitosis (26). Therefore, disruption of any of these processes will destroy cell cycle progression. In this study, we proved that inhibition of Aurora kinases caused a decrease of cdc25c, leading to an accumulation of Tyr15-phosphorylated cdc2, thus cdc2 was kept inactive. Hence cells could not enter mitosis and arrest in G2 phase. Inhibition of Aurora kinases downregulated the expression of cyclin B companied with the G2 phase arrest both in cells and SN12C xenograft tumors. Taken together, those results indicated the downregulation of cyclinB/cdc2 caused by inhibition of Aurora kinases contributed to the G2/M accumulation in ccRCC. Thus we concluded that Aurora kinases were the key regulators of the cell cycle in ccRCC.

Some reports have suggested a role of ERK1/2 signaling in G2/M checkpoint control related with cdc2-Tyr15 phosphorylation following DNA damage (27) and suppression of ERK by PD98059 treatment blocked both the inhibition of cell proliferation and the downregulation of cdc2 and cdc25c (28). The results in our present study displayed that miRNA- or VX680-mediated Aurora kinase inhibition could decrease the phosphorylation level of ERK in vitro and in vivo, so we deduced that Aurora kinases might be involved in the G2-phase arrest and cell proliferation via the induction of ERK pathway.

It has been reported that specific blockage of ERK pathway in colon tumor cells could inhibit the alterations in cell cell contact and motility that is required for metastasis (29). Our results support those findings. We observed that inhibition of Aurora kinases by miRNAs or VX680 could reduce the metastasis ability of ccRCC cells accompanied with blocking the activity of ERK.

In conclusion, in this study, we described the contribution of Aurora kinase signaling to cell proliferation, metastasis and cell cycle regulation in ccRCC. Inhibition of Aurora kinases downregulated the activity of ERK, therefore regulating the expression of the cell cycle regulator cdc25c and cdc2. Downregulation of Aurora kinases also caused the decrease of cyclin B. Hence we deduced that Aurora kinases could exerted its effect on cell cycle and proliferation through affecting the formation of cyclinB/cdc2 via ERK signal in ccRCC.

In this study, we only investigated the function of Aurora kinases on the cell cycle, proliferation, metastasis and the relationship of Aurora kinases with ERK phosphorylation in ccRCC.

Thus, although we proved that inhibition of Aurora kinases might block the ccRCC progression, and Aurora kinases might become therapy targets, more studies are needed on the complex networks of the Aurora kinases pathway and their role(s) in ccRCC.

\section{Acknowledgements}

We thank Dr Jindong Chen (Kidney Cancer Research Laboratory, Department of Urology University of Rochester Medical Center) for help in revising the manuscript. This study was supported by The National Natural Science Foundation of China (Approval number: 81102025).

\section{References}

1. Bensalah K and Patard JJ: Kidney cancer in 2010: drugs, surgery and survival in RCC. Nat Rev Urol 8: 66-68, 2010. 
2. Chowdhury $\mathrm{S}$ and Choueiri TK: Recent advances in the systemic treatment of metastatic papillary renal cancer. Expert Rev Anticancer Ther 9: 373-379, 2009.

3. Chowdhury S, Matrana MR, Tsang C, et al: Systemic therapy for metastatic non-clear-cell renal cell carcinoma: recent progress and future directions. Hematol Oncol Clin North Am 25: 853-869, 2011

4. Keen N and Taylor S: Aurora-kinase inhibitors as anticancer agents. Nat Rev Cancer 4: 927-936, 2004.

5. Tyler RK, Shpiro N, Marquez R and Eyers PA: VX-680 inhibits Aurora A and Aurora B kinase activity in human cells. Cell Cycle 6: 2846-2854, 2007.

6. Yang H, He L, Kruk P, et al: Aurora-A induces cell survival and chemoresistance by activation of Akt through a p53-dependent manner in ovarian cancer cells. Int J Cancer 119: 2304-2312, 2006.

7. Dutertre S, Cazales M, Quaranta M, et al: Phosphorylation of CDC25B by Aurora-A at the centrosome contributes to the G2-M transition. J Cell Sci 117: 2523-2531, 2004.

8. Gizatullin F, Yao Y, Kung V, et al: The Aurora kinase inhibitor VX-680 induces endoreduplication and apoptosis preferentially in cells with compromised p53-dependent postmitotic checkpoint function. Cancer Res 66: 7668-7677, 2006.

9. Merk BC, Owens JL, Lopes MB, et al: STAT6 expression in glioblastoma promotes invasive growth. BMC Cancer 11: 184, 2011.

10. Cloos J, Temmink O, Ceelen M, et al: Involvement of cell cycle control in bleomycin-induced mutagen sensitivity. Environ Mol Mutagen 40: 79-84, 2002

11. Temmink OH, Prins HJ, van Gelderop E and Peters GJ: The Hollow Fibre Assay as a model for in vivo pharmacodynamics of fluoropyrimidines in colon cancer cells. Br J Cancer 96: 61-66, 2007

12. Li Y, Zhang ZF, Chen J, et al: VX680/MK-0457, a potent and selective Aurora kinase inhibitor, targets both tumor and endothelial cells in clear cell renal cell carcinoma. Am J Transl Res 2: 296-308, 2010.

13. Huang D, Ding Y, Luo WM, et al: Inhibition of MAPK kinase signaling pathways suppressed renal cell carcinoma growth and angiogenesis in vivo. Cancer Res 68: 81-88, 2008.

14. Harrington EA, Bebbington D, Moore J, et al: VX-680, a potent and selective small-molecule inhibitor of the Aurora kinases, suppresses tumor growth in vivo. Nat Med 10 262-267, 2004.

15. Teicher BA: Newer cytotoxic agents: attacking cancer broadly. Clin Cancer Res 14: 1610-1617, 2008.

16. Suzuki T, Urano T, Miki Y, et al: Nuclear cyclin B1 in human breast carcinoma as a potent prognostic factor. Cancer Sci 98 644-651, 2007.
17. Perdiguero E and Nebreda AR: Regulation of Cdc25C activity during the meiotic G2/M transition. Cell Cycle 3: 733-737, 2004.

18. Singh SV, Herman-Antosiewicz A, Singh AV, et al: Sulforaphane-induced G2/M phase cell cycle arrest involves checkpoint kinase 2-mediated phosphorylation of cell division cycle 25C. J Biol Chem 279: 25813-25822, 2004.

19. Pearson G, Robinson F, Beers Gibson T, et al: Mitogen-activated protein (MAP) kinase pathways: regulation and physiological functions. Endocr Rev 22: 153-183, 2001.

20. Lee SJ, Park K, Ha SD, et al: Gleditsia sinensis thorn extract inhibits human colon cancer cells: the role of ERK1/2, G2/Mphase cell cycle arrest and p53 expression. Phytother Res 24: $1870-1876,2010$

21. Meraldi P, Honda R and Nigg EA: Aurora kinases link chromosome segregation and cell division to cancer susceptibility. Curr Opin Genet Dev 14: 29-36, 2004.

22. Gautschi O, Heighway J, Mack PC, et al: Aurora kinases as anticancer drug targets. Clin Cancer Res 14: 1639-1648, 2008.

23. Lee EC, Frolov A, Li R, et al: Targeting Aurora kinases for the treatment of prostate cancer. Cancer Res 66: 4996-5002, 2006.

24. Lee YM, Ting CM, Cheng YK, et al: Mechanisms of 2-methoxyestradiol-induced apoptosis and G2/M cell-cycle arrest of nasopharyngeal carcinoma cells. Cancer Lett 268 : 295-307, 2008

25. Margolis SS, Perry JA, Weitzel DH, et al: A role for PP1 in the $\mathrm{Cdc} 2 /$ Cyclin B-mediated positive feedback activation of Cdc25. Mol Biol Cell 17: 1779-1789, 2006.

26. Ray G, Dhar G, Van Veldhuizen PJ, et al: Modulation of cellcycle regulatory signaling network by 2 -methoxyestradiol in prostate cancer cells is mediated through multiple signal transduction pathways. Biochemistry 45: 3703-3713, 2006.

27. Cheng Y, Qiu F, Ye YC, et al: Oridonin induces G2/M arrest and apoptosis via activating ERK-p53 apoptotic pathway and inhibiting PTK-Ras-Raf-JNK survival pathway in murine fibrosarcoma L929 cells. Arch Biochem Biophys 490: 70-75, 2009.

28. Hsu YL, Kuo PL, Lin LT and Lin CC: Asiatic acid, a triterpene, induces apoptosis and cell cycle arrest through activation of extracellular signal-regulated kinase and p38 mitogen-activated protein kinase pathways in human breast cancer cells. J Pharmacol Exp Ther 313: 333-344, 2005.

29. Guruvayoorappan C and Kuttan G: Amentoflavone inhibits experimental tumor metastasis through a regulatory mechanism involving MMP-2, MMP-9, prolyl hydroxylase, lysyl oxidase, VEGF, ERK-1, ERK-2, STAT-1, NM23 and cytokines in lung tissues of C57BL/6 mice. Immunopharmacol Immunotoxicol 30: $711-727,2008$ 